\title{
Effect of Lycium bararum polysaccharides on methylmercury-induced abnormal differentiation of hippocampal stem cells
}

\author{
JIAN-YING TIAN ${ }^{1,2}$, WEI-WEI CHEN ${ }^{1}$, JING CUI $^{1}$, HAO WANG $^{1}$, CI CHAO $^{1}$, ZHI-YAN LU $^{1}$ and YONG-YI BI ${ }^{1}$ \\ ${ }^{1}$ Department of Health, Labor Health and Environment, School of Public Health, Wuhan University, Wuhan, Hubei 430072; \\ ${ }^{2}$ Department of Anatomy, Basic Medical School, Ningxia Medical University, Yinchuan, Ningxia 750004, P.R. China
}

Received December 16, 2014; Accepted February 4, 2016

DOI: $10.3892 /$ etm.2016.3415

\begin{abstract}
The aim of the present study was to observe the effects of a general extract of Lycium bararum polysaccharides (LBPs) on methylmercury ( $\mathrm{MeHg}$ )-induced damage in hippocampus neural stem cells (hNSCs). The hippocampal tissues of embryonic day 16 Sprague-Dawley rats were extracted for the isolation, purification and cloning of hNSCs. Following passage and proliferation for 10 days, the cells were allocated at random into the following groups: Control, LBPs, $\mathrm{MeHg}$ and $\mathrm{MeHg}+$ LBPs. MTT and microtubule-associated protein 2 (MAP-2)/glial fibrillary acidic protein/Hoechst immunofluorescence tests were performed to detect the differentiation and growth of hNSCs in the various groups. The differentiation rate of MeHg-treated hNSCs and the perimeter of MAP-2-positive neurons were $3.632 \pm 0.63 \%$ and $62.36 \pm 5.58 \mu \mathrm{m}$, respectively, significantly lower compared with the control group values of $6.500 \pm 0.81 \%$ and $166 \pm 8.16 \mu \mathrm{m}$ $(\mathrm{P}<0.05)$. Furthermore, the differentiation rate and the perimeter of MAP-2-positive neurons in LBPs groups cells was $7.75 \pm 0.59 \%$ and $253.3 \pm 11.21 \mu \mathrm{m}$, respectively, significantly higher compared with the control group $(\mathrm{P}<0.05)$. The same parameters in the $\mathrm{MeHg}+\mathrm{LBPs}$ group were $5.92 \pm 0.98 \%$ and $111.9 \pm 6.07 \mu \mathrm{m}$, respectively, significantly higher than the $\mathrm{MeHg}$ group $(\mathrm{P}<0.05)$. The astrocyte differentiation rates in the $\mathrm{MeHg}$ and $\mathrm{MeHg}+\mathrm{LBPs}$ group were $41.19 \pm 2.14$ and $34.58 \pm 1.70$, respectively $(\mathrm{P}<0.05)$. These results suggest that LBPs may promote the generation and development of new
\end{abstract}

Correspondence to: Professor Yong-Yi Bi, Department of Health, Labor Health and Environment, School of Public Health, Wuhan University, 115 Donghu Road, Wuhan, Hubei 430072, P.R. China E-mail: yongyib@yahoo.com.cn

Professor Jian-Ying Tian, Department of Anatomy, Basic Medical School, Ningxia Medical University, 692 Victory Street, Yinchuan, Ningxia 750004, P.R. China

E-mail: jianyingtian@126.com

Key words: Lycium bararum polysaccharides, methylmercury chloride, hippocampus, neural stem cells neurons and inhibit the MeHg-induced abnormal differentiation of astrocytes. Thus, LBPs may be considered to be a potential new treatment for $\mathrm{MeHg}$-induced neurotoxicity in hNSCs.

\section{Introduction}

The selective progressive reduction and deficit of neurons in the hippocampus region is closely associated with the progressive loss of event-related memory, and the major feature of Alzheimer's disease (AD) in the early stage of onset. The deficit of early hippocampal neuronal neurogenesis is the pathological basis that directly results in the loss of hippocampal functional neurons $(1,2)$. The pathogenic factors underlying hippocampal neuronal loss are complex, including such factors as heredity, environment and others $(3,4)$. In recent years, with the accelerated process of industrialization, the levels of mercury pollution have markedly increased (5-7). Due to the environmental accumulation of pollutants that are potentially harmful to human health, free mercury may be converted into the highly toxic compound methylmercury chloride $(\mathrm{MeHg})$, as a result of microbial methylation in water and soil $(8,9)$. MeHg may be absorbed from the diet, enter the blood and become distributed into all tissues, including the brain (10). Studies have shown that in humans and animals, $\mathrm{MeHg}$ may interfere with the neurogenesis and the survival of nerve cells (11-14).

Hippocampal neural stem cells (hNSCs) are able to differentiate into a number of cell types $(15,16)$. During normal nerve development, cellular differentiation is regulated by internal and external cellular factors, and the majority of cells differentiate into neurons (17). However, during a pathological state, endogenous cerebral stem cells proliferate and predominantly differentiate into glial cells, which rarely differentiate into the neurons $(18,19)$. The abnormal proliferation of NSCs, deficient neurogenesis and lack of new neurons are the primary causes underlying the reduction of cerebral hippocampal neurons and memory loss (20). Previous studies increasingly investigated the directed differentiation of NSCs $(18,19,21)$. The mechanism underlying the differentiation of stem cell division and processes that are regulated by interactions between extrinsic factors and intrinsic transcriptional cascades that may impact 
of these processes are of increasing interest (22). Our previous study (23) demonstrated that chronic exposure to MeHg may enhance the proliferation of hippocampal nerves; however, as these cells typically differentiate into the glial cells, the abnormal differentiation of neural stem cells may be crucially involved in the resulting neurogenesis deficiency, delayed neuron replenishment, reduction of cerebral hippocampal neuron mass and memory loss.

Mercury exposure may lead to inhibited neurogenesis in the hippocampal region, and the associated abnormal differentiation may be a key cause of memory loss $(11-14,24)$. However, although these previous studies have investigated the neurotoxic effects of mercury on memory, further studies are required to identify approaches to prevent the neurotoxic damage of nerve regeneration, improve the NSC living environment, restore normal cellular proliferation and differentiation and to reduce apoptosis in hippocampal NSCs.

Lycium bararum polysaccharides (LBPs) are a general extract of the primary active ingredients of the traditional Chinese medicinal plant L. bararum (25-27). Previous studies have shown that LBPs exert a variety of physiological effects, such as antioxidation, immunomodulation and anti-aging (25-27). Furthermore, LBPs have been shown to significantly protect the nerves, and may enhance the nerve regeneration (28).

The aim of the present study was to determine the impact of $\mathrm{MeHg}$ on the differentiation of hNSCs into neurons using in vitro experimental methods, and to evaluate the protective effects of LBPs towards hNSCs and neurons. The study also aimed to elucidate drugs that may prevent or treat the damage to neurogenesis caused by environmental $\mathrm{MeHg}$.

\section{Materials and methods}

Ethical approval. The present study was conducted in strict accordance with the recommendations in the Guide for the Care and Use of Laboratory Animals of the National Institutes of Health. The animal use protocol was reviewed and approved by the Institutional Animal Care and Use Committee of Wuhan University. All efforts were made to minimize the number and suffering of the animals used in this study.

Isolation, cloning, detection and amplification of rat-embryonic hNSCs. Specific pathogen-free grade Sprague-Dawley (SD) rats were provided by the Experimental Animal Center of Wuhan University (certificate no. SYXK (Su) 2007-0021; Wuhan, China). Rats were sacrificed via an overdose of $10 \%$ chloral hydrate $(0.3 \mathrm{ml} / 100 \mathrm{~g}$; v/v) via intraperitoneal injection. According to previously reported methods $(29,30)$, the embryonic day $16 \mathrm{SD}$ rat hippocampal tissues were extracted under the sterile conditions, then digested with $0.25 \%$ trypsin (SH30042.01; HyClone; GE Healthcare Life Sciences, Logan, UT, USA) for $20 \mathrm{~min}$ and uniformly pipetted. The cell suspension was filtered through a 200-mesh stainless steel mesh (Beijing Solarbio Science \& Technology Co., Ltd., Beijing, China), and centrifuged at $1,000 \mathrm{x} \mathrm{g}$ for $3 \mathrm{~min}$ at $20-22^{\circ} \mathrm{C}$ to eliminate the supernatant prior to mixing with $2 \%$ B27-containing Dulbecco's modified Eagle's medium (DMEM)/F12 (1:1) serum free medium
(SH30023.01B; HyClone. A rat-embryonic hNSC single cell suspension was obtained and seeded into a sterile $50-\mathrm{ml}$ flask $\left(4 \times 10^{5}\right.$ cells $\left./ \mathrm{ml}\right)$. A total of $20 \mathrm{ng} / \mathrm{ml}$ epidermal growth factor (EGF) and $10 \mathrm{ng} / \mathrm{ml}$ basic fibroblast growth factor (bFGF; Sigma-Aldrich, St. Louis, MO, USA) were added to the medium, making the final concentrations of the two factors up to $20 \mu \mathrm{g} / \mathrm{l}$. The flasks were then cultured at $37^{\circ} \mathrm{C}$ in $5 \%$ $\mathrm{CO}_{2}$ and saturated humidity for 5-6 days. After primary cells were cloned, the subcellular clone of hNSCs were passaged and proliferated and were identified by BrdU labeling and immunofluorescence detection with BrdU and nestin.

Briefly, the cells were fixed with $4 \%$ paraformaldehyde at room temperature for $5 \mathrm{~min}$ and washed three times with $0.01 \mathrm{~mol} / 1$ phosphate-buffered saline (PBS). Cells were permeabilized using $0.1 \%$ Triton $\mathrm{X}-100$ for $10 \mathrm{~min}$ and were subsequently washed with PBS prior to incubation with 5\% bovine serum albumin and 5\% normal goat serum for $30 \mathrm{~min}$. Following this, the cells were incubated overnight at $4{ }^{\circ} \mathrm{C}$ with primary monoclonal antibodies, including mouse anti-BrdU (MAB4072; 1:100) and mouse anti-nestin (MAB353; both 1:100; EMD Millipore). Excess primary antibody was removed by five washes with PBS and the cells were subsequently incubated with secondary antibodies for Cy3-conjugated goat anti-mouse IgG (AP124C) and FITC-conjugated goat anti-mouse IgG (AP181F; both 1:200; EMD Millipore) at $37^{\circ} \mathrm{C}$ for $1 \mathrm{~h}$, and washed with PBS. Fluorescence was detected using an Olympus BX51 fluorescence microscope (Olympus Corporation, Tokyo, Japan). In control experiments, the primary antibodies were omitted to confirm the specificity of the antibodies.

MeHg induction and LBPs intervention. In order to adjust the concentration of the third-passage hNSCs to 5 cells/100 $\mu \mathrm{l}$. the neurospheres were collected and centrifuged at $800 \mathrm{xg}$ for 5 min. Cell pellets were washed once with Hank's balanced salt solution (HBBS) and treated with $0.5 \%$ trypsin at $37^{\circ} \mathrm{C}$. DMEM/F12 supplemented with $10 \%$ fetal bovine serum was added to terminate enzymatic digestion. Digested neurospheres were centrifuged at $800 \mathrm{x} \mathrm{g}$ for $5 \mathrm{~min}$ and the dissociated cells were resuspended in DMEM/F12. An aliquot of dissociated cells was diluted with HBSS and counted using a hemocytometer (TC10; Bio-Rad Laboratories, Inc., Hercules, CA, USA) to estimate the number of cells.

Following this, 5 cells $/ 100 \mu 1$ were seeded into two 24 -well cell culture plates, which were pre-placed poly-L-lysine (PLL)-coated slides, respectively. Cells were divided into four groups, with 12 wells in each group, containing $1.5 \mathrm{ml}$ each: i) Blank control group: DMEM/F12 (1:1) serum-free medium (HyClone); ii) LBPs group: DMEM/F12 serum-free medium supplemented with $100 \mathrm{mg} / 1 \mathrm{LBPs}$ solution (Ningxia Wolfberry Biogical Food Company, Ningxia, China); iii) $\mathrm{MeHg}$ group: DMEM/F12 serum-free culture medium supplemented with $10 \mathrm{nmol} / 1 \mathrm{MeHgCl}$ solution (C1540000; Dr Ehrenstorfer GmbH, Augsburg, Germany); and iv) $\mathrm{MeHg}+$ LBPs group: DMEM/F12 serum-free medium supplemented with $10 \mathrm{nmol} / 1 \mathrm{MeHgCl}$ solution and $100 \mathrm{mg} / \mathrm{l} \mathrm{LBPs}$ solution. Culture plates were incubated at $37^{\circ} \mathrm{C}$ in $5 \% \mathrm{CO}_{2}$ and saturated humidity, and half of the culture medium was replaced every 3 days. Meanwhile, the differentiation status of the NSCs in each well was 


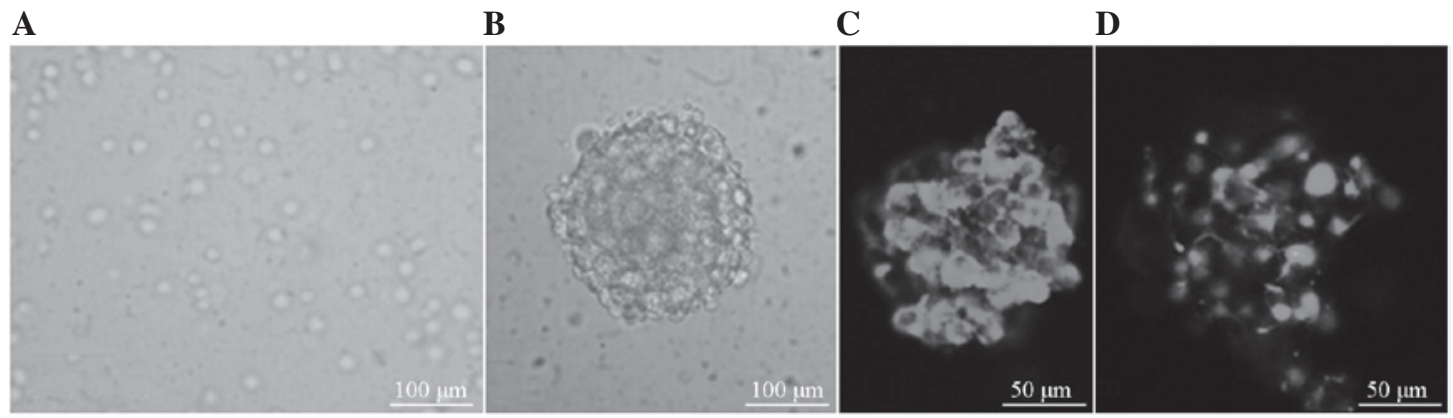

Figure 1. Hippocampus neural stem cells in primary culture and identification. (A) Isolated primary hippocampal tissue cells cultured for $2 \mathrm{~h}$, showing a single cell suspension growth. (B) Cells were cultured for 5 days, cellular proliferation and growth into small nerve cells of the sphere. (C) BrdU immunofluorescence staining, hippocampal neuron sphere was BrdU-positive. (D) Nestin immunofluorescence staining, hippocampal nerve cell spheres were Nestin-positive.

A

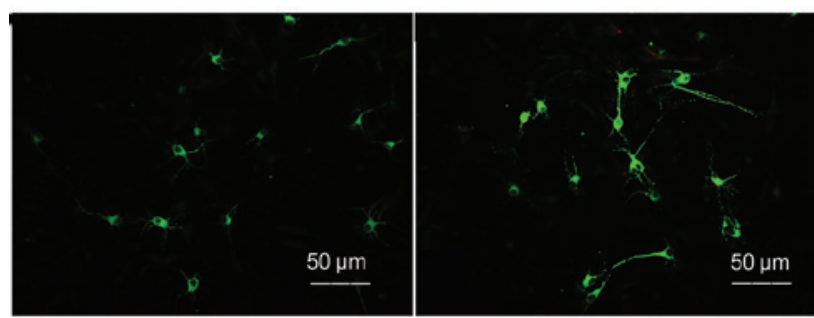

C

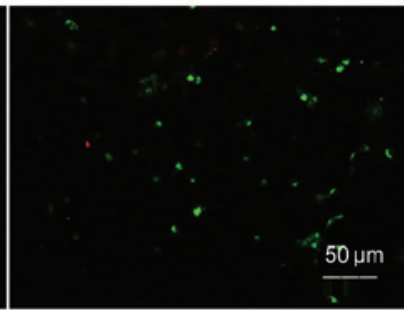

D

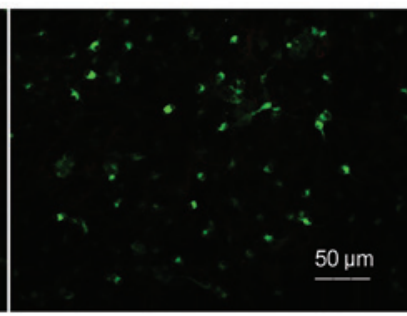

Figure 2. MAP-2-positive neurons in different culture environments (stain, MAP-2/Hoechst immunofluorescence). (A) Control group, (B) LBPs group, (C) $\mathrm{MeHg}$ group and (D) MeHg + LBPs group. MAP-2, microtubule-associated protein-2; LBPs, Lycium bararum polysaccharides; MeHg, methylmercury.

regularly observed under a Leica DM IRM inverted microscope (Leica Microsystems GmbH, Wetzlar, Germany).

Microtubule-associated protein 2 (MAP-2)/glial fibrillary acidic protein (GFAP)/Hoechst immunofluorescence detection. The culture medium of each well was discarded when differentiation was induced in the hNSCs for 10 days; which were washed with PBS ( $\mathrm{pH} 7.2)$ three times, then fixed in $1 \mathrm{ml}$ methanol at $-20^{\circ} \mathrm{C}$. Following five washes with PBS, differentiated $\mathrm{hNSCs}$ were fixed with $4 \%(\mathrm{v} / \mathrm{v})$ paraformaldehyde ( $\mathrm{pH} \mathrm{7.2)} \mathrm{at} 4^{\circ} \mathrm{C}$ for $30 \mathrm{~min}$ and washed again. Next, $10 \%$ goat serum (HyClone; $\mathrm{pH}$ 7.2) was added, and blocked with goat serum in PBS/0.3\% Triton X-100 at $4^{\circ} \mathrm{C}$ for $2 \mathrm{~h}$. Each well was then incubated with rabbit anti-MAP-2 polyclonal antibody $(1: 1,000 ;$ AB2290) and anti-mouse GFAP monoclonal antibody (1:500; MAB5628; both EMD Millipore), with a total of $200 \mu \mathrm{l}$, and agitated at room temperature for $1 \mathrm{~h}$, then incubated overnight at $4^{\circ} \mathrm{C}$. Next, the plates were agitated with $200 \mu 1$ FITC-labeled goat anti-rabbit IgG antibody $(1: 1,000$; F2761; Molecular Probes; Thermo Fisher Scientific, Inc., Waltham, MA, USA) and Alexa 568-labeled goat anti-mouse IgG antibody (1:600; A11031; Invitrogen; Thermo Fisher Scientific, Inc.) at room temperature in the dark for 2-3 h. After sufficient washing, the coverslips were mounted onto slides iwth $200 \mu 1$ Hoechst 33342 fluorescent dye (1:2,000; Calbiochem; EMD Millipore). Using an Olympus FV1000 Laser Confocal microscope and Olympus Fluoview software (Olympus Corporation), the differentiation of hNSCs into MAP-2-positive neurons and GFAP-positive astrocytes was then observed at a 495-nm excitation wavelength and 520-nm absorption wavelength, and $346 \mathrm{~nm}$ excitation wavelength and $460 \mathrm{~nm}$ absorption wavelength, respectively. Hoechst fluorescence for the nuclei were observed at $578 \mathrm{~nm}$ excitation wavelength and $608 \mathrm{~nm}$ absorption wavelength. In each group two wells were randomly selected as negative controls, with the anti-goat serum replacing the primary antibody, and all other steps performed as described above.

Image treatment. In each group an MAP-2/GFAP immunofluorescence slide had randomly selected regions undergo photography using fluorescence microscopy (magnification, x200). Bright-field images of the same field were also captured, and these were transferred into a computer. MAP-2/GFAP-positive cells were visualized under a fluorescent microscope (magnification, x200). Ten random fields of view were taken for each coverslip, and the number of nuclei and MAP-2/GFAP-incorporated cells were recorded. Corresponding Hoechst-stained total cell numbers were combined to calculate the MAP-2/GFAP-positive cell differentiation rate. All experiments were performed in triplicate. After counting and calculating the differentiation of MAP-2-positive neurons/GFAP-positive astrocytes and Hoechst total stained cells, the Leica QWin Plus 6.0 morphological analysis software (Leica Microsystems $\mathrm{GmbH}$ ) was applied to analyze the perimeter of MAP-2-positive neurons for the indirect detection of the maturity of cellular differentiation.

Statistical analysis. All the data are presented as the mean \pm standard deviation and the data underwent analysis of variance and inter-group comparison using STATA 9.0 statistical software (StataCorp LP, College Station, TX, USA). Significance of differences among mean values was determined using one-way analysis of variance. $\mathrm{P}<0.05$ was considered to indicate a statistically significant difference. 

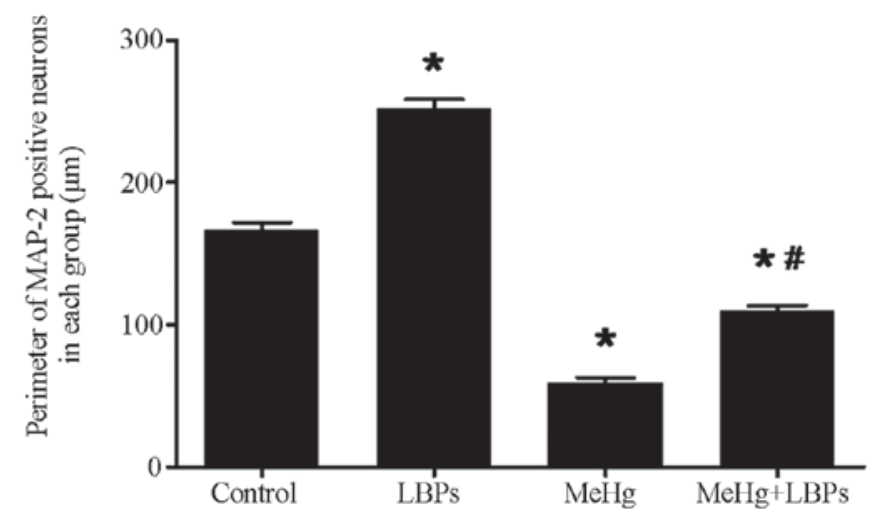

Figure 3. Perimeter of MAP-2-positive neurons in each group (observed multiples, $\mathrm{x} 200)$. Data are presented as the mean \pm standard deviation. ${ }^{*} \mathrm{P}<0.05$ vs. control; ${ }^{*} \mathrm{P}<0.05$ vs. $\mathrm{MeHg}$, as determined by Student's t-test. MAP-2, microtubule-associated protein 2; LBPs, Lycium bararum polysaccharides; $\mathrm{MeHg}$, methylmercury.

\section{Results}

Culture and identification of rat-embryonic primary and passaged hNSCs. After $2 \mathrm{~h}$ of culture, the primary hNSCs were growing singly-suspended (Fig. 1A). After 5 or 6 days, numerous suspended growing nerve spheres were observed, with smooth and clean edges, and different sizes (Fig. 1B). The primary nerve spheres could form the new nerve spheres following trypsin digestion and passaging. BrdU labeling was performed at $24 \mathrm{~h}$, and the nerve spheres were BrdU and nestin-positive, indicating that they were of embryonic origin and possessed proliferative ability, and were therefore likely to be NSCs (Fig. 1C and D).

MAP-2/Hoechst immunofluorescence detection. After the cells were cultured for 10 days, they were subjected to MAP-2/GFAP/Hoechst immunofluorescence staining to detect the neuron-differentiation and growth status of the hNSCs. The corresponding total numbers of Hoechst-stained cells were combined to calculate the neuronal differentiation rate. The MAP-2-positive neuronal differentiation rate of each group was as follows ( $n=10$ per group): Control group,

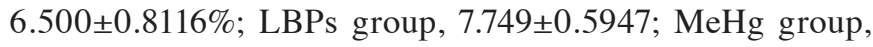
$3.632 \pm 0.6274 \%$; and $\mathrm{MeHg}+\mathrm{LBPs}$ group, $5.923 \pm 0.9868 \%$. In the control group, the MAP-2-positive neuron rate was reduced and the cell body was smaller and less mature compared with the LBPs group, the MAP-2 neuronal process length was shorter than the LBPs group, while compared with the other two groups, the cell body was bigger and the process was longer (Figs. 2A, 3 and 4A). The LBPs group exhibited the highest number of MAP-2-positive neurons, as compared with the other three groups. The neurons in this group contained large and spheric cell bodies with numerous long processes, which also intertwined among the cells, and exhibited more typical mature forms of neuron (Fig. 2B). Statistical analysis revealed that the differentiation rate of MAP-2-positive neurons in this group was the highest $(\mathrm{P}<0.05$, Fig. 4A). The number of MAP-2-positive neurons and the neuronal differentiation rate in the LBPs group were both increased, as compared with the control group $(\mathrm{P}<0.05)$. The $\mathrm{MeHg}$ group exhibited the lowest number of MAP-2-positive neurons, and

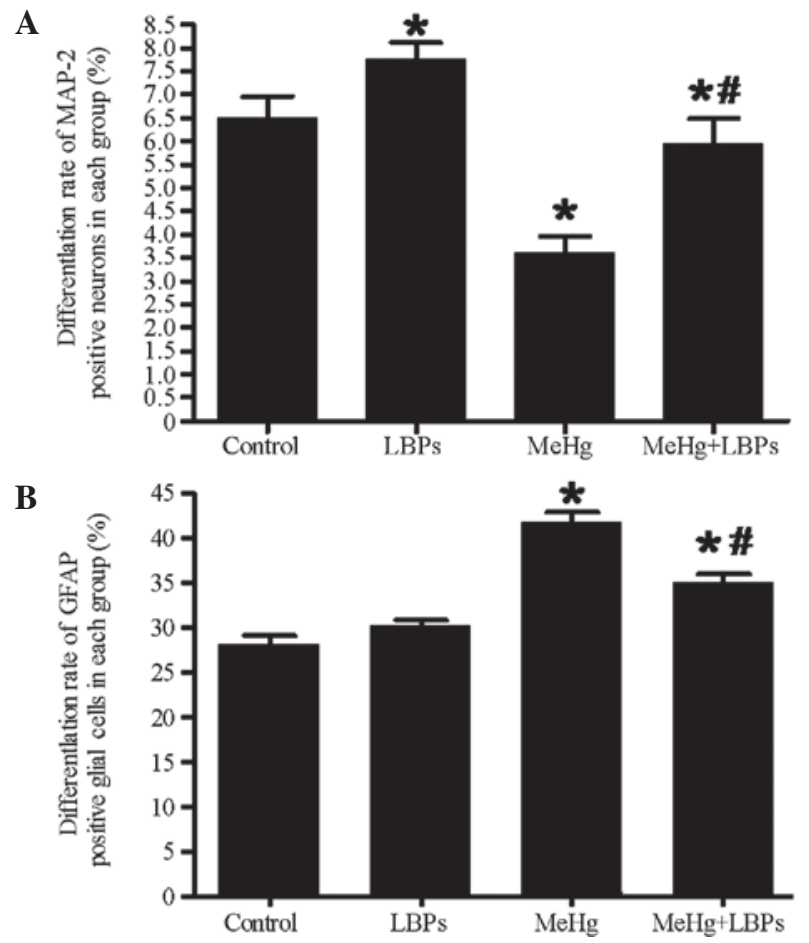

Figure 4. Differentiation rate of MAP-2 and glial fibrillary acidic protein (GFAP)-positive cells in each group.(A) Differentiation rate of MAP-2-positive neurons in each group. (B) Differentiation rate of GFAP-positive glia cells in each group. Data are presented as the mean \pm standard deviation. ${ }^{*} \mathrm{P}<0.05$ vs. control; ${ }^{\#} \mathrm{P}<0.05$ vs. $\mathrm{MeHg}$, as determined by Student's t-test. MAP-2, microtubule-associated protein 2; LBPs, Lycium bararum polysaccharides; $\mathrm{MeHg}$, methylmercury.

the majority of neurons in this group exhibited condensed cell bodies with unclear neuronal processes and irregular nuclei (Figs. 2C and 4A), indicating that $\mathrm{MeHg}$ exerted an inhibitory effect on hNSC differentiation into neurons, and also damaged hNSCs-derived neurons. In the MeHg + LBPs group, the number of MAP-2-positive neurons was increased and the cell body of neurons was bigger than the $\mathrm{MeHg}$ group, and the forms of partial neurons were considerably intact, with its processes significantly increased ( $\mathrm{P}<0.05$, Figs. 2D and 4A), indicating that LBPs was able to mitigate the suppression and damage induced by $\mathrm{MeHg}$ towards the NSCs differentiated into neurons.

GFAP/Hoechst immunofluorescence detection. The cells of the aforementioned groups additionally underwent a GFAP/Hoechst immunofluorescence assay (Fig. 5). The differentiation rate of GFAP-positive astrocytes in each of the groups was as follows ( $\mathrm{n}=10$ per group): Control group, $27.74 \pm 1.985 \%$; LBPs group, 30.18 $\pm 1.333 \%$; $\mathrm{MeHg}$ group, $41.19 \pm 2.143 \%$; and $\mathrm{MeHg}+$ LBPs group, 34.58 $\pm 1.699 \%$. The control group exhibited the lowest proportion of GFAP-positive astrocytes and the $\mathrm{MeHg}$ group exhibited the highest proportion, while the $\mathrm{MeHg}+\mathrm{LBPs}$ group was between the control and $\mathrm{MeHg}$ groups (Figs. 4B and 5). These results indicated that when $\mathrm{MeHg}$ inhibited the differentiation of NSCs into neurons, it additionally caused the differentiation of GFAP-positive astrocytes to increase. In addition, the proportion of GFAP-positive astrocytes in the $\mathrm{MeHg}+\mathrm{LBPs}$ group was decreased compared with the $\mathrm{MeHg}$ group, 
A

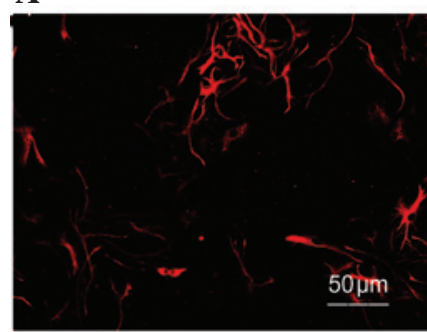

B

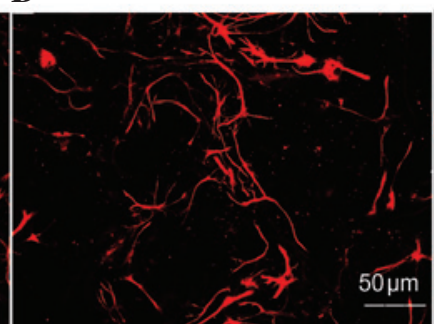

C

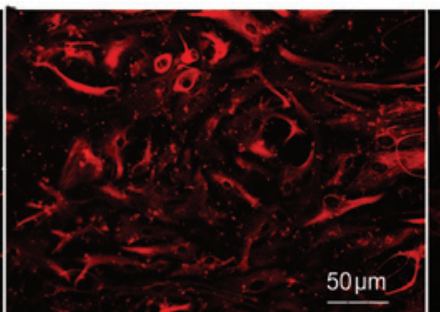

$\mathbf{D}$

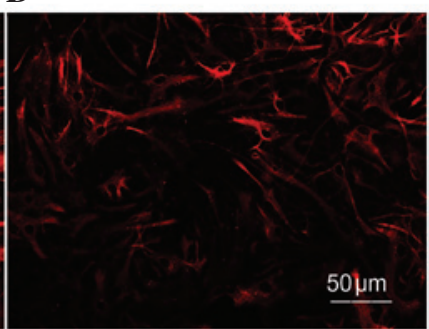

Figure 5. GFAP-positive neurons in different culture environments (GFAP/Hoechst immunofluorescence staining). (A) Control group; (B) LBPs group; (C) MeHg group; (D) MeHg + LBPs group. GFAP, glial fibrillary acidic protein; LBPs, Lycium bararum polysaccharide; MeHg, methylmercury.

indicating that LBPs were able to inhibit the MeHg-induced differentiation of NSCs into astrocytes.

\section{Discussion}

hNSCs are crucially involved in neurogenesis. hNSCs are immature nervous system cells with the capacity for self-replication and multi-directional differentiation $(21,31)$. The hippocampal dentate gyrus is a specialized region in the mammalian brain, owing to the presence of NSCs, which retain the capacity to proliferate and generate new neurons (17). The present study observed two notable features associated with hNSCs: i) Highly self-renewal capacity, the cells were able to repeat mitosis, thus generating a large number of sub-cells; and ii) under certain conditions, the cells could be induced to differentiate into neurons, glial cells and oligodendrocytes. hNSCs are crucial for the development of the embryonic nervous system, in addition to adult self-renewal and repair, learning and memory function enhancement, nerve damage processing, lost nerve cell replacement and the maintenance of the physiological functions of central nervous system.

Under normal circumstances following the loss of nerve cells lost, hNSCs are able to renew a certain number of lost neurons $(32,33)$. However, as a result of aging, various in vivo and in vitro environmental factors may affect or interfere with the hippocampal dentate gyrus neurogenic activity, and thus the neurogenesis level may gradually decrease (34). As a result of adverse external environmental factors, the internal microenvironment changes, becoming unsuitable for the survival and potential development $(35,36)$. Thus, the proliferation and differentiation of endogenous hNSCs may exhibit the exception, and the processes of generation and compensation of new neurons might be affected, the glial cells would then increase, leading to inadequate neurogenesis $(18,19)$.

A previous study showed that the absence of neurogenesis in the hippocampus was the primary cause of event-related learning memory and emotional disorders (37).

$\mathrm{MeHg}$ is an environmental toxicant that poses a cognitive degeneration risk in humans, previous studies in humans and animals have shown that $\mathrm{MeHg}$ can interfere with the processes involved in neural development, including nerve regeneration and cell survival (11-14). Thus, inhibiting the hazards of organic mercury and promoting the nerve regeneration in the hippocampus had obtained the general concerns.

Currently, stem cell transplantation had been used as a repair treatment. Coordinating stem cell self-renewal and directing differentiation are key factors for the treatment of numerous neurodegenerative diseases (38). However, the mechanism remains unclear clear, and the challenge remains of guiding the directional proliferation and differentiation of pluripotent stem cells, thus replacing missing cells in vivo and recovering functions (39).

Lycium bararum (Gouqizi, Fructus Lycii, Wolfberry) is widely used in traditional Chinese herbal medicine, food therapy and health care and is well known for improving liver function and eyesight (40). The main functional components of L. bararum include zeaxanthin and carotene, polysaccharides and small molecules such as betaine, cerebroside, beta-sitosterol, p-coumaric, and various vitamins. Previous in vivo and in vitro experiments have suggested that LBPs may have multiple targets and pharmacological effects, including neurological protection (41), antioxidation and anti-aging $(42,43)$, increasing immunity $(44)$, protecting liver cells (45) and the protection of reproductive system functions (28). Currently, increasing numbers of international studies have demonstrated the ability of LBPs to repair damaged neurons (25-27).

Previous studies have shown that LBPs is able to promote regeneration of the hippocampus and to protect hippocampal neurons $(28,46)$. Furthermore, LBPs is/are able reduce ischemia-induced necrosis and apoptosis of the retinal nerve cells, inhibit the activation of glial cells, downregulate the expression of aquaporin 4, improve oxidative stress and protect the blood-retinal barrier (41). Yu et al (47) observed that LBPs could prevent the neurotoxicity caused by the $\beta$-amyloid peptides $(A \beta)$. In addition, LBPs are able to reduce the secretion of lactate dehydrogenase and inhibit the activity of $\beta$-amyloid-like peptide protease, thus reducing the phosphorylation of c-jun-terminal kinase (JNK) and preventing the phosphorylation of neuronal protein kinase (PKR), resisting the $A \beta$-induced increase of nerve cell-endoplasmic reticulum stress levels (48). It has been reported that LBPs are able to regulate the activation of nerve microglia (brain macrophages), thus protecting retinal ganglion cells against damage cause by ocular hypertension, and exhibiting vision protective effects (49). Furthermore, LBPs are able to inhibit the cysteine-induced range of neurological damage mechanisms such as phosphorylation of tau protein, upregulation of p-ERK and p-JNK and activation of caspase-3, thus antagonize the excitotoxicity of glutamate towards the brain cortex $(50,51)$. LBPs have shown a wide range of neuroprotective effects in various neurodegenerative models. 
The present study used the embryonic rats to extract the primary hNSCs, and via in vitro culture observed the intervention effects of LBPs on MeHg-induced hNSCs damage. These results suggest that hNSCs are extremely sensitive towards the toxicity of $\mathrm{MeHg}$. $\mathrm{MeHg}$ at a $\mathrm{pM}$ concentration may decrease cellular activity, and increase the differentiation rate of astrocytes. Few NSCs differentiated into neurons, and it exhibited reduced differentiation into new neurons and astrocytes. The cellular morphology was incomplete, and the cell cycle was shortened. Following the LBPs treatment, the numbers of hNSCs that differentiated into MAP-2-positive neurons was significantly increased, and neuronal cell body was larger than the $\mathrm{MeHg}$ group. The cellular morphology of the majority of the neurons was complete, and the processes were also significantly longer, the perimeter of newborn neurons was longer compared with the $\mathrm{MeHg}$ group. Notably, the single LBPs (without MeHg) treatment promoted the differentiation of hNSCs into neurons, the differentiation rate and perimeter of newly generated neurons were higher compared with the control, indicating that LBPs were able to inhibit abnormal differentiation and alleviate new neuronal damage caused by $\mathrm{MeHg}$. Thus, the current results suggest a novel neuroprotective effect of LBPs against the reduced neurogenesis caused by $\mathrm{MeHg}$.

Highly toxic $\mathrm{MeHg}$ has been found in polluted water, soil and water, which can be a significant route of human exposure to $\mathrm{MeHg}$ (52). Eating fish is a good source of nutrition and fish oil appears to be favorable for neural development (53). Therefore, it is of interest to develop compounds that are able to bind heavy metals with high affinity and without producing toxic effects by themselves, in order to prevent developmental neurotoxicity in populations exposed to organomercurials via polluted water (54-56).

The present study showed that LBPs may be able to reduce the potential hazards associated with the mercury from fish consumption towards human health.

In addition, according to the ' 3 Rs' principles of animal experiments (reduction, replacement, and refinement), the environmental chemicals that had the developmental neurotoxicity would seriously threaten human health $(5,6) . \mathrm{MeHg}$ is able to reduce hNSC migration distance and inhibit differentiation, reducing the number of neuronal-like cells $(23,56)$. hNSCs, which grow as neurospheres may imitate proliferation, differentiation, migration and undergo caspase-dependent apoptosis in vitro, are suitable for medium-throughput screening (30) In the present study, a three-dimensional stem cell developmental neurotoxicity testing system was used to study the processes affecting NSC development and possible mechanisms underlying neurotoxic modulation. This method limits unnecessary animal experiments, and involves faster and more economical alternative screening methods to achieve the scientific purposes (30). Therefore, this cell system represents a promising approach for developmental neurotoxicity hazard identification.

In summary, hippocampal neurogenesis exhibited self-repairing potential towards the neuronal damage. The neurotoxicity of the environmental contaminant $\mathrm{MeHg}$ was associated with the inhibited regeneration of new cerebral neurons. The herbal extract LBPs was able to effectively mitigate the neurotoxic effects of $\mathrm{MeHg}$, and promote the differentiation of hNSCs into neurons, which may provide a potential approach for the recovery of hippocampus-event memory disorder.

\section{Acknowledgements}

This study was supported by the National Natural Science Fund (grant nos. 81060231 and 81160338), Ningxia Universities Key Project (grant no. NGY2011039) and the Hubei Natural Science Foundation of China (grant no. 2013CFB277).

\section{References}

1. Schaeffer EL, Novaes BA, da Silva ER, Skaf HD and Mendes-Neto AG: Strategies to promote differentiation of newborn neurons into mature functional cells in Alzheimer brain. Prog Neuropsychopharmacol Biol Psychiatry 33: 1087-1102, 2009.

2. Llorens-Martín M, Blazquez-Llorca L, Benavides-Piccione R, Rabano A, Hernandez F, Avila J and DeFelipe J: Selective alterations of neurons and circuits related to early memory loss in Alzheimer's disease. Front Neuroanat 8: 38, 2014.

3. Desplats P,Patel P, Kosberg K, Mante M,Patrick C, Rockenstein E, Fujita M, Hashimoto M and Masliah E: Combined exposure to Maneb and Paraquat alters transcriptional regulation of neurogenesis-related genes in mice models of Parkinson's disease. Mol Neurodegener 7: 49, 2012.

4. Ding D, Moskowitz SI, Li R, Lee SB, Esteban M, Tomaselli K, Chan J and Bergold PJ: Acidosis induces necrosis and apoptosis of cultured hippocampal neurons. Exp Neurol 162: 1-12, 2000.

5. Boischio A: Developmental neurotoxicity: Methylmercury and prenatal exposure protection in the context of the Minamata Convention. Rev Panam Salud Publica 38: 243-247, 2015.

6. Sheehan MC, Burke TA, Navas-Acien A, Breysse PN, McGready J and Fox MA: Global methylmercury exposure from seafood consumption and risk of developmental neurotoxicity: a systematic review. Bull World Health Organ 92: 254-269F, 2014.

7. Chen CY, Driscoll CT, Lambert KF, Mason RP and Sunderland EM: Connecting mercury science to policy: From sources to seafood.Rev Environ Health 31: 17-20, 2016.

8. Graham AM, Aiken GR and Gilmour CC: Dissolved organic matter enhances microbial mercury methylation under sulfidic conditions. Environ Sci Technol 46: 2715-2723, 2012.

9. Graham AM, Aiken GR and Gilmour CC: Effect of dissolved organic matter source and character on microbial $\mathrm{Hg}$ methylation in Hg-S-DOM solutions. Environ Sci Techno 147: 5746-5754, 2013 .

10. Clarkson TW, Vyas JB and Ballatori N: Mechanisms of mercury disposition in the body. Am J Ind Med 50: 757-764, 2007.

11. Burke K, Cheng Y, Li B, Petrov A, Joshi P, Berman RF, Reuhl KR and DiCicco-Bloom E: Methylmercury elicits rapid inhibition of cell proliferation in the developing brain and decreases cell cycle regulator, cyclin E. Neurotoxicology 27: 970-981, 2006.

12. Falluel-Morel A, Sokolowski K, Sisti HM, Zhou X, Shors TJ and Dicicco-Bloom E: Developmental mercury exposure elicits acute hippocampal cell death, reductions in neurogenesis and severe learning deficits during puberty. J Neurochem 103: 1968-1981, 2007.

13. Haase H, Engelhardt G, Hebel S and Rink L: Mercuric ions inhibit mitogen-activated protein kinase dephosphorylation by inducing reactive oxygen species. Toxicol Aplli Pharmacol 250: 78-86, 2011.

14. Sokolowski K, Falluel-Morel A, Zhou X and DiCicco-Bloom E: Methylmercury (MeHg) elicits mitochondrial-dependent apoptosis in developing hippocampus and acts at low exposures. Neurotoxicology 32: 535-544, 2011.

15. Kino T: Stress, glucocorticoid hormones, and hippocampal neural progenitor cells: implications to mood disorders. Front Physiol 6: 230, 2015.

16. Guo J, Wang J, Liang C, Yan J, Wang Y, Liu G, Jiang Z, Zhang L, Wang X, Wang Y, et al: proNGF inhibits proliferation and oligodendrogenesis of postnatal hippocampal neural stem/progenitor cells through p75NTR in vitro. Stem Cell Res 11: 874-887, 2013.

17. Cameron HA and Mckay RD: Adult neurogenesis produces a large pool of new granule cells in the dentate gyrus. J Comp Neurol 435: 406-417, 2001. 
18. Ozeki A, Suzuki K, Suzuki M, Ozawa H and Yamashita S: Acceleration of astrocytic differentiation in neural stem cells surviving X-irradiation. Neuroreport 23: 290-293, 2012.

19. Domowicz MS, Henry JG, Wadlington N, Navarro A, Kraig RP and Schwartz NB: Astrocyte precursor response to embryonic brain injury. Brain Res 1389: 35-49, 2011.

20. Zhang J, Ji F, Liu Y, Lei X, Li H, Ji G, Yuan Z and Jiao J: Ezh2 regulates adult hippocampal neurogenesis and memory. J Neurosci 34: 5184-5199, 2014.

21. Christie KJ, Emery B, Denham M, Bujalka H, Cate HS and Turnley AM: Transcriptional regulation and specification of neural stem cells. Adv Exp Med Biol 786: 129-155, 2013.

22. Hodge RD, Nelson BR, Kahoud RJ, Yang R, Mussar KE, Reiner SL and Hevner RF: Tbr2 is essential for hippocampal lineage progression from neural stem cells to intermediate progenitors and neurons. J Neurosci 32: 6275-6287, 2012.

23. Tian J, Luo Y, Chen W, Yang S, Wang H, Cui J, Lu Z, Lin Y and Bi Y: $\mathrm{MeHg}$ Suppressed neuronal potency of hippocampal NSCs contributing to the puberal spatial memory deficits. Biol Trace Elem Res: Jan 8, 2016 (Epub ahead of print).

24. Sokolowski K, Obiorah M, Robinson K, McCandlish E, Buckley B and DiCicco-Bloom E: Neural stem cell apoptosis after low-methylmercury exposures in postnatal hippocampus produce persistent cell loss and adolescent memory, deficits.Dev Neurobiol 73: 936-949, 2013.

25. Lam CS, Tipoe GL, So KF and Fung ML: Neuroprotective mechanism of Lycium barbarum polysaccharides against hippocampal-dependent spatial memory deficits in a rat model of obstructive sleep apnea. PLoS One 10: e0117990, 2015

26. Liu L, Lao W, Ji QS, Yang ZH, Yu GC and Zhong JX: Lycium barbarum polysaccharides protected human retinal pigment epithelial cells against oxidative stress-induced apoptosis. Int J Ophthalmol 8: 11-6, 2015.

27. Cheng J, Zhou ZW, Sheng HP, He LJ, Fan XW, He ZX, Sun T, Zhang $\mathrm{X}$, Zhao RJ, Gu L et al: An evidence-based update on the pharmacological activities and possible molecular targets of Lycium barbarum polysaccharides. Drug Des Devel Ther 9: 33-78, 2014.

28. Lau BW, Lee JC, Li Y, Fung SM, Sang YH, Shen J, Chang RC and So KF: Polysaccharides from wolfberry prevents corticosterone-induced inhibition of sexual behavior and increases neurogenesis. PLoS One 7: e33374, 2012.

29. Seibenhener ML and Wooten MW: Isolation and culture of hippocampal neurons from prenatal mice. J Vis Exp 65: 3634, 2012

30. Moors M, Rockel TD, Abel J, Cline JE, Gassmann K, Schreiber T, Schuwald J, Weinmann N and Fritsche E: Human neurospheres as three-dimensional cellular systems for developmental neurotoxicity testing. Environ Health Perspect 117: 1131-1138, 2009.

31. Piatti VC, Espósito MS and Schinder AF: The timing of neuronal development in adult hippocampal neurogenesis. Neuroscientist 12: 463-468, 2006

32. Rolls A, Shechter R, London A, Ziv Y, Ronen A, Levy R and Schwartz M: Toll-like receptors modulate adult hippocampal neurogenesis. Nat Cell Biol 9: 1081-1088, 2007.

33. Llorens-Martín M, Rábano A and Ávila J: The ever-changing morphology of hippocampal granule neurons in physiology and pathology. Front Neurosci 9: 526, 2016.

34. Snyder JS, Hong NS, Mcdonald RJ and Wojtowicz JM: A role for adult neurogenesis in spatial long-term memory. Neuroscience 130: 843-852, 2005

35. Ryan SM and Nolan YM: Neuroinflammation negatively affects adult hippocampal neurogenesis and cognition: Can exercise compensate? Neurosci Biobehav Rev 61: 121-131, 2016.

36. Jinno S: Aging affects new cell production in the adult hippocampus: A quantitative anatomic review. J Chem Neuroanat S08 91-0618(15)00094-0, 2015.

37. Mielke JG and Mealing GA: Cellular distribution of the nicotinic acetylcholine receptor alpha7 subunit in rat hippocampus. Neurosci Res 65: 296-306, 2009.

38. Januschke J and Näthke I: Stem cell decisions: A twist of fate or a niche market? Semin Cell Dev Biol 34: 116-123, 2014.
39. Abdel-Salam OM: Stem cell therapy for Alzheimer's disease. CNS Neurol Disord Drug Targets 10: 459-485, 2011.

40. Paul Hsu CH, Nance DM and Amagase H: A meta-analysis of clinical improvements of general well-being by a standardized Lycium barbarum. J Med Food 15: 1006-1014, 2012.

41. Yang D, Li SY, Yeung CM, Chang RC, So KF, Wong D and Lo AC: Lycium barbarum extracts protect the brain from blood-brain barrier disruption and cerebral edema in experimental stroke. PLoS One 7: e33596, 2012.

42. He M, Pan H, Chang RC, So KF, Brecha NC and Pu M Activation of the Nrf2/HO-1 antioxidant pathway contributes to the protective effects of Lycium barbarum polysaccharides in the rodent retina after ischemia-reperfusion-induced damage. PLoS One 9: e84800, 2014.

43. Chang RC and So KF: Use of anti-aging herbal medicine, Lycium barbarum, against aging-associated diseases. What do we know so far? Cell Mol Neurobiol 28: 643-652, 2008.

44. Yang M, Ding J, Zhou X, Zhang X, Tao H, Wang Y and Li G: Effects of Lycium barbarum polysaccharides on neuropeptide $Y$ and heat-shock protein 70 expression in rats exposed to heat Biomed Rep 2: 687-692, 2014.

45. Xiao J, Xing F, Huo J, Fung ML, Liong EC, Ching YP, Xu A, Chang RC, So KF and Tipoe GL: Lycium barbarum polysaccharides therapeutically improve hepatic functions in non-alcoholic steatohepatitis rats and cellular steatosis model. Sci Rep 4: 5587, 2014.

46. Rui C, Yuxiang L, Yinju H, Qingluan Z, Yang W, Qipeng Z, Hao W, Lin M, Juan L, Chengjun Z, et al: Protective effects of Lycium barbarum polysaccharide on neonatal rat primary cultured hippocampal neurons injured by oxygen-glucose deprivation and reperfusion. J Mol Histol 43: 535-542, 2012.

47. Yu MS, Leung SK, Lai SW, Che CM, Zee SY, So KF, Yuen WH and Chang RC: Neuroprotective effects of anti-aging oriental medicine Lycium barbarum against beta-amyloid peptide neurotoxicity. Exp Gerontol 40: 716-727, 2005.

48. Yu MS, Ho YS, So KF, Yuen WH and Chang RC: Cytoprotective effects of Lycium barbarum against reducing stress on endoplasmic reticulum. Int J Mol Med 17: 1157-1161, 2006.

49. Mi XS, Feng Q, Lo AC, Chang RC, Lin B, Chung SK and So KF: Protection of retinal ganglion cells and retinal vasculature by Lycium barbarum polysaccharides in a mouse model of acute ocular hypertension. PLoS One 7: e45469, 2012.

50. Ho YS, Yu MS, Yang XF, So KF, Yuen WH and Chang RC: Neuroprotective effects of polysaccharides from wolfberry, the fruits of Lycium barbarum, against homocysteine-induced toxicity in rat cortical neurons. J Alzheimers Dis 19: 813-827, 2010.

51. Ho YS, Yu MS, Yik SY, So KF, Yuen WH and Chang RC: Polysaccharides from wolfberry antagonizes glutamate excitotoxicity in rat cortical neurons. Cell Mol Neurobiol 29: 1233-1244, 2009.

52. Clarkson TW and Strain JJ: Nutritional factors may modify the toxic action of methyl mercury in fish-eating populations. J Nutr 133: 1539S-1543S, 2003.

53. Byelashov OA, Sinclair AJ and Kaur G: Dietary sources, current intakes, and nutritional role of omega-3 docosapentaenoic acid. Lipid Technol 27: 79-82, 2015.

54. Ayyathan DM, Chandrasekaran $\mathrm{R}$ and Thiagarajan $\mathrm{K}$ : Neuroprotective effect of Brahmi, an ayurvedic drug against oxidative stress induced by methyl mercury toxicity in rat brain mitochondrial-enriched fractions. Nat Prod Res 29: 1046-1051, 2015.

55. Kaur P, Aschner M and Syversen T: Biochemical factors modulating cellular neurotoxicity of methylmercury. J Toxicol 2011: 721987, 2011

56. Wagner C, Vargas AP, Roos DH, Morel AF, Farina M, Nogueira CW, Aschner M and Rocha JB: Comparative study of quercetin and its two glycoside derivatives quercitrin and rutin against methylmercury $(\mathrm{MeHg})$-induced ROS production in rat brain slices. Arch Toxicol 84: 89-97, 2010 